\title{
Maximal Operator in Variable Exponent Generalized Morrey Spaces on Quasi-metric Measure Space
}

\author{
Vagif S. Guliyev and Stefan G. Samko
}

\begin{abstract}
We consider generalized Morrey spaces $\mathcal{L}^{p(\cdot), \varphi(\cdot)}(X)$ on quasimetric measure spaces $X, d, \mu$, in general unbounded, with variable exponent $p(x)$ and a general function $\varphi(x, r)$ defining the Morrey-type norm. No linear structure of the underlying space $X$ is assumed. The admission of unbounded $X$ generates problems known in variable exponent analysis. We prove the boundedness results for maximal operator known earlier only for the case of bounded sets $X$. The conditions for the boundedness are given in terms of the so called supremal inequalities imposed on the function $\varphi(x, r)$, which are weaker than Zygmund-type integral inequalities often used for characterization of admissible functions $\varphi$. Our conditions do not suppose any assumption on monotonicity of $\varphi(x, r)$ in $r$.
\end{abstract}

Mathematics Subject Classification. 42B25, 42B35, 46E30.

Keywords. Maximal operator, variable exponent generalized Morrey space, quasi-metric measure space.

\section{Introduction}

Variable exponent function spaces is now an extensively developed field with two books $[8,10]$ having already appeared on variable exponent Lebesgue and Sobolev spaces. Nowadays many problems for various operators of harmonic analysis are solved in these spaces including a number weight problem. There are also various advances for variable exponent Morrey function spaces, but to a less extent than for Lebesgue spaces.

For classical Morrey spaces we refer to the books $[15,28,34]$ and the recent survey paper [32]; in the last reference an information on various versions of variable exponent Morrey function spaces may be found. The Morrey spaces $\mathcal{L}^{p(\cdot), \lambda(\cdot)}(X)$ with variable exponents $\lambda(\cdot)$ and $p(\cdot)$ in the Euclidean setting were introduced and studied in $[3,12,25,26,31]$. 
General version $\mathcal{L}^{p(\cdot), \varphi(\cdot)}(\Omega)$, of such Morrey spaces, $\Omega \subseteq \mathbb{R}^{n}$ with variable exponent were introduced and studied in [16]. In [17] the results of [16] on the boundedness of maximal, singular and potential operators were extended to unbounded sets in $\mathbb{R}^{n}$.

A success in developing variable exponent analysis in the Euclidean setting was naturally accompanied by generalizations of the case where the underlying space was a quasi-metric measure space $(X, d, \mu)$ mainly with doubling measure. A progress was achieved mainly for bounded sets in $X$. With respect to variable exponent Lebesgue spaces on quasi-metric measure spaces we refer to $[4,5,13,14,20-22,24]$, see all references therein.

In particular, the maximal and singular operators were considered in $[21,24]$. Results on potential operators and Sobolev embeddings may be found in $[4,5,13,14,20,22]$.

Variable exponent Morrey spaces on bounded spaces of homogeneous type and maximal and singular operators in such spaces were studied in $[25,26]$.

Variable exponent Campanato spaces on bounded homogeneous spaces were studied in [33], where there was proved their equivalence to variable exponent Morrey spaces or variable exponent Hölder spaces in the undercritical and overcritical cases, respectively.

Note that, contrast to the case of constant exponents, admission of unbounded sets for variable exponents generate difficulties well known to researchers in this topic. In the Euclidean case they were overcome for variable Lebesgue spaces in [9] (see also more details in the books [8,10], and for variable Morrey spaces in [17]).

Variable exponent Lebesgue spaces on unbounded quasi-metric measure spaces were considered in [1,27]. They appeared in [27] where the weighted maximal operator was studied when $p(x)$ is constant at infinity. In the very recent paper [1] the maximal operator was studied without weights but under a more general decay condition at infinity.

In this paper, we prove theorems on the boundedness of the maximal operator

$$
M f(x)=\sup _{r>0} \frac{1}{\mu(B(x, r))} \int_{B(x, r)}|f(y)| d \mu(y)
$$

within the frameworks of variable exponent Morrey spaces $\mathcal{L}^{p(\cdot), \varphi(\cdot)}(X)$ on unbounded quasi-metric measure spaces, which extends the corresponding result of [17] obtained in the Euclidean case.

The paper is organized as follows. In Sect. 2 we provide necessary definitions and tools from the theory of quasi-metric measure spaces. In Sect. 3 we give necessary background from variable exponent Lebesgue spaces and prove some auxiliary statements for such spaces on infinite quasi-metric measure sets. Section 4 contains the definition of a generalized Morrey spaces adjusted for underlying sets with general structure and some auxiliary results. Finally, Sect. 5 contains the main statements and their proof. 


\section{On Quasi-metric Measure Spaces}

\subsection{Basic Definitions and Some Properties}

In the sequel, $(X, d, \mu)$ denotes a quasi-metric space with the (quasi) metric $d$, satisfying the condition

$$
d(x, y) \leq k[d(x, z)+d(z, y)], \quad k \geq 1
$$

and Borel regular measure $\mu$.

Everywhere in the sequel $\chi_{E}(x)$ stands for the characteristic function of a set $E \subseteq X$ and

$$
\ell=\operatorname{diam} X .
$$

We refer to $[11,23]$ for the basics on metric measure spaces. By

$$
B(x, r)=\{y \in X: d(x, y)<r\}
$$

we denote a ball in $X$. We assume that the following standard conditions are satisfied:

1. all the balls $B(x, r)$ are measurable,

2. the space $C(X)$ of uniformly continuous functions on $X$ is dense in $L^{1}(X, \mu)$.

The doubling condition

$$
\mu B(x, 2 r) \leq C_{\mathrm{dbl}} \times \mu B(x, r),
$$

where $C_{\mathrm{dbl}} \geq 1$ does not depend on $r>0$ and $x \in X$, is known to play a big role in the theory of quasi-metric measure spaces. We will also refer sometimes to the following counterpart of this condition:

$$
\mu B(x, 2 r) \geq \mathbb{C} \times \mu B(x, r), \quad r>0 \quad \text { with } \quad \mathbb{C}>1
$$

(uniformly in $x$ and $r$ ).

The conditions

$$
\mu\left(B(x, r)<c_{1} r^{n} .\right.
$$

and

$$
\mu B(x, r) \geq c_{0} r^{N}
$$

imposed sometimes on the measure $\mu$ are known as the upper and lower Ahlfors conditions; the first one is also referred to as the growth condition. We find it important to emphasize that in the case of unbounded sets $X$, it is natural to assume that Ahlfors conditions may hold with different exponents for small and large $r$, in general. An illustrating example is

$$
\begin{aligned}
(X, d, \mu) & =\left(\mathbb{R}^{n}, d, \mu\right) \quad \text { with } \quad d(x, y)=|x-y| \quad \text { and } \\
\mu(E) & =\int_{E}(1+|x|)^{\lambda} d x, \quad \lambda>0 .
\end{aligned}
$$

In this case

$$
\mu B(x, r) \approx C(x) r^{n} \quad \text { as } \quad r \rightarrow 0,
$$

but

$$
\mu B(x, r) \approx C(x) r^{n+\lambda} \quad \text { as } \quad r \rightarrow \infty
$$


Thus, the following definition turns to be natural.

Definition 2.1. Let $(X, d \mu)$ be unbounded. We say that the measure $\mu$ satisfies upper Ahlfors condition with the exponents $n_{0}>0, n_{\infty}>0$, if

$$
\mu(B(x, r)) \leq c r^{n(r)}, \quad r>0, \quad n(r)=\left\{\begin{array}{ll}
n_{0}, & \text { if } 0<r \leq 1 \\
n_{\infty}, & \text { if } r \geq 1
\end{array} .\right.
$$

and lower Ahlfors condition with the exponents $N_{0}>0, N_{\infty}>0$, if

$$
\mu(B(x, r)) \geq c r^{N(r)}, \quad r>0, \quad N(r)=\left\{\begin{array}{ll}
N_{0}, & \text { if } 0<r \leq 1 \\
N_{\infty}, & \text { if } r \geq 1
\end{array} .\right.
$$

Remark 2.1. In the estimates (2.6) and (2.7) one can obviously take arbitrary bounded values of the exponents $n(r)$ and $N(r)$, when $0<a \leq r \leq b<\infty$, so that one can replace the piecewise constant exponents, for instance in (2.6) by an arbitrary bounded function $n(r)$, stabilizing to $n_{0}$ and $n_{\infty}$ as $r \rightarrow 0$ and $r \rightarrow \infty$, respectively. This remains equivalent if $n(r)$ stabilizes under the logdecay condition. One can go even further and admit, at the least for the upper Ahlfors condition, the exponents $n(r)$ oscillating at infinity between two different positive constants. However, we do not touch such cases in this paper.

Sometimes we will use the following assumptions:

$$
\inf _{x \in X} \mu B(x, r)>0
$$

and

$$
\sup _{x \in X} \mu B(x, r)<\infty
$$

for any fixed $r>0$.

Lemma 2.1. If the property (2.8) holds, then

$(2.2) \Longrightarrow$ the lower Ahlfors condition (2.5) for $r \rightarrow 0$

(with the exponent $N=\log _{2} C_{\mathrm{dbl}}$ ). In case $X$ is infinite, if (2.9) holds, then

$(2.2) \Longrightarrow$ the upper Ahlfors condition (2.4) for $r \rightarrow \infty$

(with the same exponent). Similarly, if (2.9) holds, then

$(2.3) \Longrightarrow$ the upper Ahlfors condition (2.4) for $r \rightarrow 0$

(with the exponent $n=\log _{2} \mathbb{C}$ ). In the case $X$ is infinite, if (2.8) holds, then

$(2.3) \Longrightarrow$ the upper Ahlfors condition (2.5) for $r \rightarrow \infty$

(with the same exponent).

Proof. The statements of the lemma are in fact known. In the case of (2.2) they follow from the known estimate

$$
\mu B(x, \varrho) \leq C\left(\frac{\varrho}{r}\right)^{N} \mu B(x, r), \quad N=\log _{2} C_{\mathrm{dbl}},
$$

for $0<r \leq \varrho$, where $C>0$ does not depend on $r, \varrho$ and $x$, which is derived from (2.2) by iteration. Similarly from (2.3) one can obtain that

$$
\mu B(x, \varrho) \geq C\left(\frac{\varrho}{r}\right)^{n} \mu B(x, r), \quad n=\log _{2} \mathbb{C},
$$

for $0<r \leq \varrho$, which yields the statements for $(2.3)$. 


\section{Auxiliary Results for Variable Exponent Lebesgue Spaces}

\subsection{Preliminaries: Basic Definitions}

Let $p(\cdot)$ be a measurable function on $X$ with values in $[1, \infty)$. We assume that $1 \leq p_{-} \leq p(x) \leq p_{+}<\infty$, but in most cases suppose that $1<p_{-} \leq$ $p(x) \leq p_{+}<\infty$. By $L^{p(\cdot)}(X)$ we denote the space of all measurable functions $f(x)$ on $X$ such that

$$
I_{p(\cdot)}(f)=\int_{X}|f(x)|^{p(x)} d \mu(x)<\infty .
$$

Equipped with the norm

$$
\|f\|_{p(\cdot)}=\inf \left\{\eta>0: I_{p(\cdot)}\left(\frac{f}{\eta}\right) \leq 1\right\},
$$

this is a Banach function space. By $p^{\prime}(x)=\frac{p(x)}{p(x)-1}, x \in X$, we denote the conjugate exponent.

We use the following notation:

$$
p_{-}=p_{-}(X)=\inf _{x \in X} p(x), \quad p_{+}=p_{+}(X)=\sup _{x \in X} p(x),
$$

$\mathcal{P}(X)$ is the set of bounded measurable functions $p: X \rightarrow[1, \infty)$;

$\mathcal{P}^{\log }(X)$ is the set of exponents $p \in \mathcal{P}(X)$ satisfying the local logcondition

$$
|p(x)-p(y)| \leq \frac{A_{p}}{-\ln d(x, y)}, \quad d(x, y) \leq \frac{1}{2} \quad x, y \in X,
$$

where $A=A(p)>0$ does not depend on $x, y$;

$\mathcal{A}^{\log }(X)$ is the set of bounded exponents $\alpha: X \rightarrow \mathbb{R}$ satisfying the condition $(3.1)$;

$\mathbb{P}^{\log }(X)$ is the set of exponents $p \in \mathcal{P}^{\log }(X)$ with $1<p_{-} \leq p_{+}<\infty$;

for $X$ which may be unbounded, by $\mathcal{P}_{\infty}(X), \mathcal{P}_{\infty}^{\log }(X), \mathbb{P}_{\infty}^{\log }(X), \mathcal{A}_{\infty}^{\log }(X)$ we denote the subsets of the above sets of exponents satisfying the decay condition:

$$
|p(x)-p(\infty)| \leq \frac{A_{\infty}}{\ln \left(2+d\left(x, x_{0}\right)\right)} \quad x \in X,
$$

where $x_{0}$ is any fixed point in $X$.

We will also use the decay condition in the form

$$
|p(x)-p(\infty)| \leq \frac{A_{\infty}}{\ln \mu B\left(x, d\left(x, x_{0}\right)\right)} \quad x \in X, \quad d\left(x, x_{0}\right) \geq 2 .
$$

Remark 3.1. It is easy to see that (3.2) implies (3.3) if $\mu$ satisfies the growth condition (2.4) at infinity, and (3.3) implies (3.2) if $\mu$ satisfies lower Ahlfors condition at infinity.

We will use the following boundedness result for the maximal operator proved in [1, Corollary 1.6] (in [1] a more general result was obtained, we use it in the form we need in this paper).

Theorem 3.1. Let $X$ be doubling and $p \in \mathbb{P}_{\infty}^{\log }(X)$. Then

$$
\|M f\|_{L^{p(\cdot)}(X)} \leq C\|f\|_{L^{p(\cdot)}(X)} .
$$




\subsection{Estimates for $\left\|\chi_{B(x, r)}\right\|_{L^{p(\cdot)}(X)}$ on Unbounded Quasi-metric Measure Spaces}

The estimate

$$
\left\|\chi_{B(x, r)}\right\|_{p(\cdot)} \leq c[\mu B(x, r)]^{\frac{1}{p(x)}}
$$

for bounded open sets $X$ was proved in the setting of quasi-metric measure space in [22], see also an alternative proof in [3, Lemma 6] under the assumptions that the measure $\mu$ satisfies the lower Alhfors condition (2.5) and $p(\cdot)$ satisfies the log-condition.

The following lemma provides an estimate for $\left\|\chi_{B(x, r)}\right\|_{p(\cdot)}$ suitable for unbounded sets $X$; in the Euclidean setting it was given in [10, Corollary 4.5.9].

For an exponent $p(x)$ defined on an unbounded set $X$ and satisfying the decay condition (3.2) we use the notation

$$
p_{r}(x)=\left\{\begin{array}{ll}
p(x), & 0<r \leq 1, \\
p(\infty), & r \geq 1
\end{array}, \quad x \in X .\right.
$$

Theorem 3.2. Let the measure $\mu$ be doubling and condition (2.3) be satisfied. If $p \in \mathcal{P}^{\log }(X)$ and the decay condition in the form (3.3). Then

$$
\left\|\chi_{B(x, r)}\right\|_{p(\cdot)} \leq c[\mu B(x, r)]^{\frac{1}{p_{r}(x)}}
$$

for all $x \in X$ and $r>0$.

Proof. We wish to show that

$$
\int_{B(x, r)} \frac{d \mu(y)}{[\mu(B(x, r))]^{\frac{p(y)}{p_{r}(x)}}} \leq C \text { for all } r>0 \text { and } x \in X .
$$

For $0<r \leq 1$ this is known, being contained in (3.4) (note that the case $r \leq 1$ does not require $X$ to be bounded, since we always have $\frac{1}{|\ln | d(x, y)||} \leq \frac{1}{|\ln r|}$ in this case).

So let $r \geq 1$. We have to show that the function

$$
\widetilde{F}(x, r):=\int_{B(x, r)} \frac{d \mu(y)}{[\mu(B(x, r))]^{\frac{p(y)}{p(\infty)}}}
$$

is bounded. We fix any point $x_{0} \in X$. It is obvious that it suffices to show the boundedness of the function

$$
\widetilde{F}(x, r):=\int_{\left\{y \in B(x, r): d\left(y, x_{0}\right)>1\right\}} \frac{d \mu(y)}{[\mu B(x, r)]^{\frac{p(y)}{p(\infty)}}}
$$

is bounded. Let first $d\left(x, x_{0}\right) \geq 2 k r$, where $k$ is the constant from the triangle inequality, so that $d\left(y, x_{0}\right)>\frac{1}{k} d\left(x, x_{0}\right)-d(x, y)>r$. Then

$$
|p(y)-p(\infty)| \ln \mu B(x, r) \leq|p(y)-p(\infty)| \ln \mu B\left(x, d\left(x, x_{0}\right)\right) \leq C
$$

by $(3.3)$, which yields

$$
[\mu(B(x, r))]^{\frac{p(y)}{p(\infty)}} \geq C \mu(B(x, r)
$$


and provides the boundedness of $\widetilde{F}(x, r)$.

Let now $d\left(x, x_{0}\right)<2 k r$. Then $B(x, r) \subset B\left(x_{0},(2 k+1) r\right)$, so that

$$
\widetilde{F}(x, r) \leq \int_{1<d\left(y, x_{0}\right)<(2 k+1) r} \frac{d \mu(y)}{\mu B(x, r)^{\frac{p(y)}{p(\infty)}}},
$$

where we proceed as follows:

$$
\widetilde{F}(x, r) \leq \sum_{j} \int_{L_{j}(r)} \frac{d \mu(y)}{[\mu B(x, r)]^{\frac{p(y)}{p(\infty)}}},
$$

where we denoted

$L_{j}(r):=\left\{y: d\left(y, x_{0}\right)>1\right\} \cap\left\{y: 2^{-j-1}(2 k+1) r<d\left(y, x_{0}\right)<2^{-j}(2 k+1) r\right\}$, $j=0,1,2, \ldots$ Hence,

$$
\widetilde{F}(x, r) \leq \sum_{j} \int_{L_{j}(r)} \frac{d \mu(y)}{\left[\mu B \left(x,\left(2^{j} d\left(y, x_{0}\right) /(2 k+1)\right]^{\frac{p(y)}{p(\infty)}}\right.\right.} .
$$

By the property (2.3) we then have

$$
\begin{aligned}
\widetilde{F}(x, r) & \leq \sum_{j} \mathbb{C}^{-j \frac{p_{-}}{p(\infty)}} \int_{L_{j}(r)} \frac{d \mu(y)}{\left[\mu B\left(x, \frac{d\left(y, x_{0}\right)}{2 k+1}\right)\right]^{\frac{p(y)}{p(\infty)}}} \\
& \leq \sum_{j} \mathbb{C}^{-j \frac{p_{-}}{p(\infty)}} \int_{L_{j}(r)} \frac{d \mu(y)}{\left[\mu B\left(x, d\left(y, x_{0}\right)\right)\right]^{\frac{p(y)}{p(\infty)}}},
\end{aligned}
$$

where the doubling condition was used in the last inequality. Then

$$
\widetilde{F}(x, r) \leq \sum_{j} \mathbb{C}^{-j \frac{p-}{p(\infty)}} \int_{2^{-j-1}(2 k+1) r<d\left(y, x_{0}\right)<2^{-j}(2 k+1) r} \frac{d \mu(y)}{\mu B\left(x, d\left(y, x_{0}\right)\right)}
$$

by the decay condition. We use the doubling condition again and obtain $\int_{2^{-j-1}(2 k+1) r<d\left(y, x_{0}\right)<2^{-j}(2 k+1) r} \frac{d \mu(y)}{\mu B\left(x, d\left(y, x_{0}\right)\right)} \leq \frac{\mu B\left(x, r 2^{-j}(2 k+1) r\right)}{\mu B\left(x, 2^{-j-1}(2 k+1) r\right)} \leq C$, which completes the proof.

\section{Variable Exponent Generalized Morrey Spaces}

\subsection{The Case of Variable Exponent Classical Morrey Spaces}

Variable exponent Morrey spaces were introduced in the Euclidean setting in [3] and on quasi-metric measure spaces in $[25,26]$. The definition below follows the same approach, up to notation.

Let $\lambda(x)$ be a measurable function on $X$ with values in $[0,1]$. The variable Morrey space $\mathcal{L}^{p(\cdot), \lambda(\cdot)}(X)$ is introduced via the norm

$$
\|f\|_{\mathcal{L}^{p(\cdot), \lambda(\cdot)}(X)}=\sup _{x \in X, t>0} \mu B(x, t)^{-\frac{\lambda(x)}{p(x)}}\left\|f \chi_{B(x, t)}\right\|_{L^{p(\cdot)}(X)} .
$$


In [3] such spaces in the Euclidean case $X=\Omega \subset \mathbb{R}^{n}$ were also defined by means of the norm

$$
\sup _{x \in X, r>0}\left\|\mu B(x, r)^{-\frac{\lambda(x)}{p(\cdot)}} f \chi_{B(x, r)}\right\|_{p(\cdot)} .
$$

This norm is equivalent to the above norm when $X$ is bounded and $p$ satisfies the log-condition.

In [25] the following theorem for bounded sets $X$ was proved.

Theorem 4.1. Let $X$ be bounded and $p \in \mathbb{P}^{\log }(X)$ and $\lambda(x) \geq 0, \sup _{x \in X} \lambda(x)$ $<1$. Then the maximal operator $M$ is bounded in $\mathcal{L}^{p(\cdot), \lambda(\cdot)}(X)$.

A version of such a result for $X, d, \mu$ with doubling condition replaced by the growth condition on the measure $\mu$ was proved in [26, Theorem 3.4].

\subsection{Variable Exponent Generalized Morrey Spaces}

To avoid confusion in notation, we use the letter $\mathcal{M}$ for generalized Morrey spaces defined by a general function $\varphi(x, r)$, see $(4.1)$, and we keep the letter $\mathcal{L}$ for the classical version of Morrey spaces when $\varphi(x, r)=\mu B(x, r)^{\frac{\lambda(x)}{p(x)}}$.

Everywhere in the sequel the functions $\varphi(x, r), \varphi_{1}(x, r)$ and $\varphi_{2}(x, r)$ are non-negative measurable functions on $X \times \mathbb{R}_{+}$.

Definition 4.1. Let $p \in \mathcal{P}(X)$. The generalized Morrey space $\mathcal{M}^{p(\cdot), \varphi(\cdot)}(X)$ is defined by the norm

$$
\|f\|_{\mathcal{M}^{p(\cdot), \varphi(\cdot)}}=\sup _{x \in X, r>0} \frac{\|f\|_{L^{p(\cdot)}(B(x, r))}}{\varphi(x, r)} .
$$

Everywhere in the sequel we assume that

$$
\inf _{x \in X} \varphi(x, r)>0
$$

for every $r>0$, which makes the space $\mathcal{M}^{p(\cdot), \varphi(\cdot)}(X)$ nontrivial.

Note that by the definition of the norm in $L^{p(\cdot)}$ we have

$\|f\|_{\mathcal{M}^{p(\cdot), \varphi(\cdot)}}=\sup _{x \in X, r>0} \inf \left\{\eta=\eta(x, r): \int_{X}\left|\frac{f(y) \chi_{B(x, r)}(y)}{\eta \varphi(x, r)}\right|^{p(y)} d \mu(y) \leq 1\right\}$.

The spaces $\mathcal{M}^{p(\cdot), \varphi(\cdot)}(X)$ contain in particular classical Morey spaces with different measuring of the "Morrey-type regularity" for small and large values of $r$, i.e., the spaces

$$
\mathcal{L}^{p(\cdot), \lambda(\cdot), \lambda_{\infty}(\cdot)}(X)
$$

defined by the norm

$$
\begin{aligned}
& \|f\|_{\mathcal{L}^{p(\cdot), \lambda(\cdot), \lambda_{\infty}(\cdot)}} \\
& \quad=\sup _{x \in X}\left(\sup _{0<r<1} r^{-\frac{\lambda(x)}{p(x)}}\left\|f \chi_{B(x, r)}\right\|_{L^{p(\cdot)}(X)}+\sup _{r>1} r^{-\frac{\lambda_{\infty}(x)}{p(x)}}\left\|f \chi_{B(x, r)}\right\|_{L^{p(\cdot)}(X)}\right),
\end{aligned}
$$

corresponding to the choice $\varphi(x, r)= \begin{cases}r^{\lambda(x)}, & r \leq 1, \\ r^{\lambda_{\infty}(x)}, & r \geq 1\end{cases}$ 
The norm (4.3) prompts us also to introduce another norm

$\|f\|_{\mathcal{M}^{p(\cdot), \varphi(\cdot)}}^{*}=\sup _{x \in X, r>0} \inf \left\{\eta=\eta(x, r): \frac{1}{\varphi(x, r)^{p(x)}} \int_{X}\left|\frac{f(y)}{\eta}\right|^{p(y)} d \mu(y) \leq 1\right\}$.

The norms $\|f\|_{\mathcal{M}^{p(\cdot), \varphi(\cdot)}}$ and $\|f\|_{\mathcal{M}^{p(\cdot), \varphi(\cdot)}}^{*}$ are non-equivalent in general and we denote by

$$
\mathcal{M}_{*}^{p(\cdot), \varphi(\cdot)}(X)
$$

the space of functions $f$ with finite norm $\|f\|_{\mathcal{M}^{p(\cdot), \varphi(\cdot)}}^{*}$. In Lemma 4.2 we provide some conditions under which the spaces $\mathcal{M}_{*}^{p(\cdot), \varphi(\cdot)}(X)$ and $\mathcal{M}^{p(\cdot), \varphi(\cdot)}(X)$ coincide.

In the spirit of (4.3), we may also introduce the corresponding versions $\mathcal{L}_{*}^{p(\cdot), \lambda(\cdot)}(X)$ of classical type Morrey spaces, defined similarly to (4.3) by the norm

$$
\|f\|_{\mathcal{L}^{p(\cdot), \lambda(\cdot)}}^{*}=\sup _{x \in X, r>0} \inf \left\{\eta=\eta(x, r): \frac{1}{r^{\lambda(x)}} \int_{B(x, r)}\left|\frac{f(y)}{\eta}\right|^{p(y)} d \mu(y) \leq 1\right\} .
$$

We single out the case where

$$
\varphi(x, r) \equiv \text { const for } r \geq 1,
$$

i.e., the case where the "Morrey regularity" is measured only for small $r$. The Morrey space with the function $\varphi(x, r)$ satisfying the property (4.6), might be called locally introduced Morrey space.

In Lemma 4.2 we use the log-condition in the form

$$
|p(x)-p(y)| \times|\ln \varphi(x, r)| \leq C \quad \text { for } \quad x, y \in X \quad \text { with } \quad d(x, y) \leq r \leq 1,
$$

where $C$ does not depend on $x, y$ and $r$. The following lemma provides a sufficient condition for the validity of (4.7).

Lemma 4.1. The condition (4.7) is satisfied, if $p \in \mathcal{P}(X)$, the function $\varphi$ is bounded, fulfills the condition (4.2) and

$$
\varphi(x, r) \geq \frac{C_{0}}{\ln ^{a} \frac{1}{r}}, \quad \text { for some } \quad a>0
$$

in a neighborhood $0 \leq r \leq \varepsilon$ of the origin.

Proof. It suffices to consider the case where $\varphi(x, r) \leq \frac{1}{2}$ [otherwise there is nothing to prove in (4.7)]. We may assume that $C_{0}=1$ and consider small $r$. Then

$$
\ln \frac{1}{\varphi(x, r)} \leq a \ln \ln \frac{1}{r} \leq a \ln \frac{1}{r} \leq a \ln \frac{1}{d(x, y)},
$$

so that the usual log-condition for $p$ implies (4.7).

Lemma 4.2. Let $p \in \mathcal{P}$ and $\varphi(x, r)$ fulfill the conditions (4.6) and (4.7). Then the norms $\|f\|_{\mathcal{M}^{p(\cdot), \varphi(\cdot)}}$ and $\|f\|_{\mathcal{M}^{p(\cdot), \varphi(\cdot)}}^{*}$ are equivalent. 
Proof. It suffices to prove that $c_{1} \varphi(x, r)^{p(x)} \leq \varphi(x, r)^{p(y)} \leq c_{2} \varphi(x, r)^{p(x)}$, which follows from (4.7) under the condition (4.6).

\section{Main Results}

In this section, in both the theorems it is assumed that the space $(X, d, \mu)$ is doubling and the condition (2.3) is satisfied.

\subsection{Estimation of $\|M f\|_{L^{p(\cdot)}(B(x, t))}$}

Theorem 5.1. Let $\mu$ be doubling and satisfy the growth condition (2.4) for $r \rightarrow \infty$ and let $p \in \mathbb{P}_{\infty}^{\log }(X)$. Then

$$
\|M f\|_{L^{p(\cdot)}(B(x, t))} \leq C \mu B(x, t)^{\frac{1}{p_{t}(x)}} \sup _{r>t} \mu B(x, r)^{-\frac{1}{p_{r}(x)}}\|f\|_{L^{p(\cdot)}(B(x, r))}, \quad t>0
$$

for every $f \in L^{p(\cdot)}(X)$, where $C$ does not depend on $f, x \in X$ and $t$.

Proof. We split $f$ as

$$
\begin{aligned}
& f=f_{1}+f_{2}, \quad f_{1}(y)=f(y) \chi_{B(x, 2 k t)}(y), \\
& f_{2}(y)=f(y) \chi_{X \backslash B(x, 2 k t)}(y), \quad t>0 .
\end{aligned}
$$

where $k$ is the constant from the triangle inequality (2.1).

Estimation of $M f_{1}$ By Theorem 3.1 we have

$$
\left\|M f_{1}\right\|_{L^{p(\cdot)}(B(x, t))} \leq\left\|M f_{1}\right\|_{L^{p(\cdot)}(X)} \leq C\left\|f_{1}\right\|_{L^{p(\cdot)}(X)}=C\|f\|_{L^{p(\cdot)}(B(x, 2 k t))},
$$

where $C$ does not depend on $f$. To unify this estimate in the sequel with the estimate for $\left\|M f_{2}\right\|_{L^{p(\cdot)}(B(x, t))}$, we transform the obtained estimate to

$$
\left\|M f_{1}\right\|_{L^{p(\cdot)}(B(x, t))} \leq C \mu B(x, t)^{\frac{1}{p_{t}(x)}} \sup _{r>t} \mu B(x, r)^{-\frac{1}{p_{r}(x)}}\|f\|_{L^{p(\cdot)}(B(x, r))}
$$

by the obvious inequality $1 \leq \mu B(x, t)^{\frac{1}{p_{t}(x)}} \sup _{r>t} \mu B(x, r)^{-\frac{1}{p_{r}(x)}}$ and monotonicity of $\|f\|_{L^{p(\cdot)}(B(x, t))}$ with respect to $t$.

Estimation of $M f_{2}$ For

$$
M f_{2}(y)=\sup _{r>0} \frac{1}{\mu(B(y, r))} \int_{B(y, r) \cap^{\mathrm{C}}(B(x, 2 k t))}|f(z)| d \mu(z)
$$

we observe that

$$
B(y, r) \cap^{c}(B(x, 2 k t)) \subseteq B(x, 2 k r) \quad \text { for every } \quad y \in B(x, t)
$$

whenever

$$
B(y, r) \cap{ }^{\mathrm{C}}(B(x, 2 k t)) \neq \emptyset .
$$

Note that the condition (5.6) implies $r>t$. Indeed, if $z \in B(y, r) \cap^{\mathrm{C}}$ $(B(x, 2 k t))$, then $r>d(y, z) \geq \frac{1}{k} d(x, z)-d(x, y)>2 t-t=t$.

Consequently, for $z \in B(y, r) \cap^{\mathrm{C}}(B(x, 2 k t))$ we have $d(x, z) \leq k d(y, z)+$ $k d(x, y)<k t+k r<2 k r$, which proves $(5.5)$. 
By (5.5) we get

$$
\begin{aligned}
M f_{2}(y) & \leq \sup _{r>t} \frac{1}{\mu(B(x, r))} \int_{B(x, 2 k r)}|f(z)| d \mu(z) \\
& \leq \sup _{r>2 k t} \frac{C}{\mu(B(x, r))} \int_{B(x, r)}|f(z)| d \mu(z) \\
& \leq \sup _{r>t} \frac{C}{\mu(B(x, r))} \int_{B(x, r)}|f(z)| d \mu(z)
\end{aligned}
$$

by the doubling condition, for all $y \in B(x, t)$.

Hence by Hölder inequality and Theorem 3.2 we obtain

$$
\begin{aligned}
M f_{2}(y) & \leq \sup _{r>t} \frac{C}{\mu(B(x, r))}\|f\|_{L^{p(\cdot)}(B(x, r))}\|1\|_{L^{p^{\prime}(\cdot)}(B(x, r))} \\
& \leq C \sup _{r>t}\|f\|_{L^{p(\cdot)}(B(x, r))} \mu B(x, r)^{-1+\frac{1}{p_{r}^{\prime}(x)}} \\
& =C \sup _{r>t}\|f\|_{L^{p(\cdot)}(B(x, r))} \mu B(x, r)^{\frac{1}{p_{r}(x)}},
\end{aligned}
$$

Theorem 3.2 is applicable by the conditions of our theorem, because the assumption of Theorem 3.2 on the validity of the decay condition in the form (3.3) holds, see Remark 3.1.

Thus, the function $M f_{2}(y)$, with fixed $x$ and $t$, is dominated by the expression not depending on $y$. Then we integrate the obtained estimate for $M f_{2}(y)$ in $y$ over $B(x, t)$, we get

$$
\left\|M f_{2}\right\|_{L^{p(\cdot)}(B(x, t))} \leq C\|1\|_{L^{p(\cdot)}(B(x, t))} \sup _{r>t}\|f\|_{L^{p(\cdot)}(B(x, r))} \mu B(x, r)^{-\frac{1}{p_{r}(x)}} .
$$

Then we apply Theorem 3.2 again and obtain the estimate for $\left\|M f_{2}\right\|_{L^{p(\cdot)}(B(x, t))}$ as in (5.4) for $\left\|M f_{1}\right\|_{L^{p(\cdot)}(B(x, t))}$. Gathering the estimates, we arrive at (5.1).

\subsection{Preliminaries on Supremal Operator}

By $L^{\infty}\left(\mathbb{R}_{+}, v\right)$ we denote the space of all functions $g(t), t>0$ with finite norm $\|g\|_{L^{\infty}\left(\mathbb{R}_{+}, v\right)}=\sup _{t>0} v(t) g(t)$. Let $\mathfrak{M}^{+}\left(\mathbb{R}_{+}\right)$be the set of all nonnegative Lebesgue-measurable functions on $\mathbb{R}_{+}$and $\mathfrak{M}^{+}\left(\mathbb{R}_{+} ; \uparrow\right)$ be the cone of all non-decreasing functions in $\mathfrak{M}^{+}\left(\mathbb{R}_{+}\right)$and

$$
\mathcal{A}=\left\{\varphi \in \mathfrak{M}^{+}\left(\mathbb{R}_{+} ; \uparrow\right): \lim _{t \rightarrow 0+} \varphi(t)=0\right\} .
$$

Let $u$ be a continuous non-negative function on $\mathbb{R}_{+}$. We define the supremal operator $\bar{S}_{u}$ for $g \in \mathfrak{M}\left(\mathbb{R}_{+}\right)$by

$$
\left(\bar{S}_{u} g\right)(t):=\|u g\|_{L^{\infty}(t, \infty)}, \quad t \in \mathbb{R}_{+} .
$$

The following theorem was proved in $[6]$. 
Theorem 5.2. Let $v_{1}, v_{2}$ be non-negative measurable functions with $0<$ $\left\|v_{1}\right\|_{L^{\infty}(t, \infty)}<\infty$ for every $t>0$ and let $u$ be a continuous non-negative function on $\mathbb{R}$. Then the operator $\bar{S}_{u}$ is bounded from $L^{\infty}\left(\mathbb{R}_{+}, v_{1}\right)$ to $L^{\infty}\left(\mathbb{R}_{+}, v_{2}\right)$ on the cone $\mathcal{A}$ if and only if

$$
\left\|v_{2} \bar{S}_{u}\left(\left\|v_{1}\right\|_{L^{\infty}(\cdot, \infty)}^{-1}\right)\right\|_{L_{\infty}\left(\mathbb{R}_{+}\right)}<\infty
$$

\subsection{Theorem on the Boundedness}

Theorem 5.3. Let the space $(X, d, \mu)$ and the exponent p satisfy the assumptions of Theorem 5.1. If

$$
\sup _{t>r} \frac{\inf _{t<s<\infty} \varphi_{1}(x, s)}{\mu B(x, t)^{\frac{1}{p_{t}(x)}}} \leq C \frac{\varphi_{2}(x, r)}{\mu B(x, r)^{\frac{1}{p_{r}(x)}}},
$$

where $C$ does not depend on $x$ and $r$. Then the maximal operator $M$ is bounded from the space $\mathcal{M}^{p(\cdot), \varphi_{1}(\cdot)}(X)$ to the space $\mathcal{M}^{p(\cdot), \varphi_{2}(\cdot)}(X)$.

Proof. For the norm

$$
\|M f\|_{\mathcal{M}^{p(\cdot), \varphi_{2}(\cdot)}(X)}=\sup _{x \in X, t>0} \varphi_{2}^{-1}(x, t)\|M f\|_{L^{p(\cdot)}(B(x, t))}
$$

by Theorems 5.1 and 5.2 we obtain

$$
\begin{aligned}
& \|M f\|_{\mathcal{M}^{p(\cdot), \varphi_{2}(\cdot)}(X)} \\
& \quad \leq C \sup _{x \in X, t>0} \varphi_{2}^{-1}(x, t) \mu B(x, t)^{\frac{1}{p_{t}(x)}} \sup _{r>t} \mu B(x, r)^{-\frac{1}{p_{r}(x)}\|f\|_{L^{p(\cdot)}(B(x, r))}} \\
& \quad \leq C \sup _{x \in X, t>0} \varphi_{1}^{-1}(x, t)\|f\|_{L^{p(\cdot)}(B(x, t))}=C\|f\|_{\mathcal{M}^{p(\cdot), \varphi_{1}(\cdot)}(X)}
\end{aligned}
$$

by (5.9), which completes the proof.

Remark 5.1. Supremal estimates for the maximal operator in generalized Morrey spaces with constant $p$ and in the Euclidean setting were obtained in $[2]$.

In the case of constant exponents $p$ boundedness results for the maximal operator in classical Morrey spaces go back to [7]. For Morrey spaces with constant $p$ but a general function $\varphi(x, r)$ defining the Morrey space, such results under these or those assumptions were obtained in $[18,19,29,30]$.

Corollary 5.1. Let $(X, d, \mu)$ and the exponent $p$ satisfy the assumptions of Theorem 5.1. Under the conditions

$$
0 \leq \lambda(x) \leq \min \left(1, \frac{p(x)}{p(\infty)}\right)
$$

the maximal operator $M$ is bounded in the space $\mathcal{L}^{p(\cdot), \lambda(\cdot)}(X)$.

Proof. Apply Theorem 5.3 with $\varphi_{1}(x, r)=\varphi_{2}(x, r)=\mu B(x, r)^{\frac{\lambda(x)}{p(x)}}$.

Corollary 5.1 covers, in particular, the case of quasi-metric measure spaces $(X, d, \mu)$ with constant dimension:

$$
\mu B(x, r) \approx r^{a}
$$


for some positive $a$ (i.e., there exist positive constants $a, c_{1}$ and $c_{2}$ such that $c_{1} r^{a} \leq \mu B(x, r) \leq c_{2} r^{a}$ for all $x \in X$ and $\left.r \rightarrow 0\right)$. Besides the Euclidean case, an important example is a Carleson curve on the plane or fractal curves with constant dimension.

The statement of Corollary 5.1 was proved in [3] for bounded sets $X$, where the last of the conditions in (5.10) does not appear.

\section{Acknowledgements}

The authors would like to express their gratitude to the referees for his (her) very valuable comments and suggestions. The research of V. Guliyev was partially supported by the grant of Science Development Foundation under the President of the Republic of Azerbaijan, Grant EIF-2013-9(15)-46/10/1 and by the grant of Presidium Azerbaijan National Academy of Science 2015. The research of S. Samko was partially supported by the Grant 1501-02732 of Russian Fund of Basic Research and by the grant of TUBITAK BIDEB Project No.: B.14.2.TBT.0.06.01.03.220.01-29104 during his visit to Dumlupinar University, Turkey, in April of 2014.

\section{References}

[1] Adamowicz, T., Harjulehto, P., Hästö, P.: Maximal operator in variable exponent Lebesgue spaces on unbounded quasimetric measure spaces. J. Math. Scand. 116(1), 5-22 (2015). http://www.helsinki.fi/pharjule/varsob/pdf/ maximal-submitted

[2] Akbulut, A., Guliyev, V.S., Mustafayev, R.: On the boundedness of the maximal operator and singular integral operators in generalized morrey spaces. Math. Bohem. 13(1), 27-43 (2012)

[3] Almeida, A., Hasanov, J., Samko, S.: Maximal and potential operators in variable exponent Morrey spaces. Georgian Math. J. 15(2), 195-208 (2008)

[4] Almeida, A., Samko, S.: Embeddings of variable Hajlasz-Sobolev spaces into Hölder spaces of variable order. J. Math. Anal. Appl. 353, 489-496 (2009)

[5] Almeida, A., Samko, S.: Fractional and hypersingular operators in variable exponent spaces on metric measure spaces. Meditter. J. Math. 6, 215-232 (2009)

[6] Burenkov, V.I., Gogatishvili, A., Guliyev, V.S., Mustafayev, R.C.: Boundedness of the fractional maximal operator in local Morrey-type spaces. Complex Var. Elliptic Equ. 55(8-10), 739-758 (2010)

[7] Chiarenza, F., Frasca, M.: Morrey spaces and Hardy-Littlewood maximal function. Rend. Math. 7, 273-279 (1987)

[8] Cruz-Uribe, D., Fiorenza, A.: Variable Lebesgue Spaces. Foundations and Harmonic Analysis, Birkhäuser (2013)

[9] Cruz-Uribe, D., Fiorenza, A., Neugebauer, C.J.: The maximal function on variable $L^{p}$-spaces. Ann. Acad. Sci. Fenn. Math. 28, 223-238 (2003)

[10] Diening, L., Harjulehto, P., Hästö, P., Růžička, M.: Lebesgue and Sobolev spaces with variable exponents. In: Lecture Notes in Mathematics, vol. 2017. Springer, Berlin (2011) 
[11] Edmunds, D.E., Kokilashvili, V., Meskhi, A.: Bounded and compact integral operators. In: Mathematics and its Applications, vol. 543. Kluwer, Dordrecht (2002)

[12] Fan, X.: The regularity of Lagrangians $f(x, \xi)=|\xi|^{\alpha(x)}$ with Hölder exponents $\alpha(x)$. Acta Math. Sin. (N.S.) 12(3), 254-261 (1996)

[13] Futamura, T., Harjulehto, P., Hästö, P., Mizuta, Y., Shimomura, T.: Variable exponent spaces on metric measure spaces. In: More Progresses in Analysis, Proceedings of ISAAC-5, Catania, 2005, vol. 126, pp. 107-121. World Scientific (2009)

[14] Futamura, T., Mizuta, Y., Shimomura, T.: Sobolev embeddings for variable exponent Riesz potentials on metric spaces. Ann. Acad. Sci. Fenn. Math. 31(2), 495-522 (2006)

[15] Giaquinta, M.: Multiple Integrals in the Calculus of Variations and Non-linear Elliptic Systems. Princeton University Press, Princeton (1983)

[16] Guliyev, V.S., Hasanov, J., Samko, S.: Boundedness of the maximal, potential and singular operators in the generalized variable exponent Morrey spaces. Math. Scand. 107, 285-304 (2010)

[17] Guliyev, V.S., Samko, S.: Maximal, potential and singular operators in the generalized variable exponent Morrey spaces on unbounded sets. J. Math. Sci. 193(2), 228-248 (2013)

[18] Guliyev, V.S.: Integral operators on function spaces on homogeneous groups and on domains in $R^{n}$ (in Russian). Ph.D. thesis, Doctor's degree, Moscow, Steklov Math. Inst. (1994)

[19] Guliyev, V.S.: Function spaces, integral operators and two weighted inequalities on homogeneous groups. Some applications (in Russian). Casioglu, Baku (1999)

[20] Hajibayov, M., Samko, S.: Generalized potentials in variable exponent Lebesgue spaces on homogeneous spaces. Math. Nachr. 284(1), 53-66 (2011)

[21] Harjulehto, P., Hästö, P., Pere, M.: Variable exponent Lebesgue spaces on metric spaces: the Hardy-Littlewood maximal operator. Real Anal. Exch. 30(1), 87-103 (2004)

[22] Harjulehto, P., Hästö, P., Latvala, V.: Sobolev embeddings in metric measure spaces with variable dimension. Math. Z. 254(3), 591-609 (2006)

[23] Heinonen, J.: Lectures on analysis on metric spaces. In: Universitext. Springer, New York (2001)

[24] Khabazi, M.: The maximal operator in spaces of homogenous type. Proc. A. Razmadze Math. Inst. 138, 17-25 (2005)

[25] Kokilashvili, V., Meskhi, A.: Boundedness of maximal and singular operators in Morrey spaces with variable exponent. Armen. J. Math. 1(1), 18-28 (2008)

[26] Kokilashvili, V., Meskhi, A.: Maximal functions and potentials in variable exponent Morrey spaces with non-doubling measure. Complex Var. Elliptic Equ. 55(8-10), 923-936 (2010)

[27] Kokilashvili, V., Samko, N., Samko, S.: The maximal operator in weighted variable Lebesgue spaces on metric spaces. Georgian Math. J. 15(4), 683-712 (2008)

[28] Kufner, A., John, O., Fučik, S.: Function spaces, pp. $454+$ XV. Noordhoff International Publishing, Prague (1977) 
[29] Mizuhara, T.: Boundedness of some classical operators on generalized Morrey spaces. In: Igari, S. (ed) Harmonic Analysis, pp. 183-189. Springer, Berlin (1991). (ICM 90 Satellite Proceedings)

[30] Nakai, E.: Hardy-Littlewood maximal operator, singular integral operators and the Riesz potentials on generalized Morrey spaces. Math. Nachr. 166, 95-103 (1994)

[31] Ohno, T.: Continuity properties for logarithmic potentials of functions in Morrey spaces of variable exponent. Hiroshima Math. J. 38(3), 363-383 (2008)

[32] Rafeiro, H., Samko, N., Samko, S.: Morrey-Campanato spaces: an overview. In: Karlovich, Y.I., Rodino, L., Silbermann, B., Spitkovsky, I.M. (eds.) Operator Theory, Pseudo-differential Equations, and Mathematical Physics The Vladimir Rabinovich Anniversary Volume, Operator Theory: Advances and Applications, vol. 228, pp. 293-323. Springer, Birkhäuser (2013)

[33] Rafeiro, H., Samko, S.: Variable exponent Campanato spaces. J. Math. Sci. (N. Y.) 172(1), 143-164 Problems in mathematical analysis. No. 51 (2011)

[34] Taylor, M.E.: Tools for PDE, volume 81 of Mathematical Surveys and Monographs. American Mathematical Society, Providence (2000). Pseudodifferential operators, paradifferential operators, and layer potentials

\author{
Vagif S. Guliyev \\ Institute of Mathematics and Mechanics \\ B. Vahabzade 9
}

AZ1141 Baku

Azerbaijan

e-mail: vagif@guliyev.com

and

Department of Mathematics

Ahi Evran University

Bagbasi Campus

40100 Kirsehir

Turkey

Stefan G. Samko

Universidade do Algarve

Estrada da Penha

8005-139 Faro

Portugal

e-mail: ssamko@ualg.pt

Received: November 4, 2014.

Revised: March 7, 2015.

Accepted: March 19, 2015. 